\title{
Undercarboxylated osteocalcin inhibits the early differentiation of osteoclast mediated by Gprc6a
}

\author{
Hailong Wang Equal first author, 1 , Jinqiao Li ${ }^{\text {Equal first author, } 1,2}{ }^{2}, Z_{\text {ihan Xu }}{ }^{1}$, Feng Wu ${ }^{1}$, Hongyu Zhang ${ }^{1}$, Chao Yang ${ }^{1}$, Jian Chen $^{1,3}$ \\ , Bai Ding ${ }^{1}$, Xiukun Sui ${ }^{1}$, Zhifeng Guo ${ }^{1}$, Yinghui Li ${ }^{\text {Corresp., } 1}$, Zhongquan Dai ${ }^{\text {Corresp. } 1}$ \\ ${ }^{1}$ State Key Laboratory of Space Medicine Fundamentals and Application, China Astronaut Research and Training Center, Beijing, China \\ 2 Space Engineering University, Beijing, China \\ 3 Department of Orthopaedics, Sir Run Run Shaw Hospital, School of Medicine, Zhejiang University, Hangzhou, China \\ Corresponding Authors: Yinghui Li, Zhongquan Dai \\ Email address: yinghuidd@vip.sina.com, daizhq77@163.com
}

Osteocalcin (OCN) was the most abundant noncollagen protein and considered as an endocrine factor. However, the functions of Undercarboxylated osteocalcin (UCOCN) on osteoclast and bone resorption are not well understood. In the present study, preosteoclast RAW264.7 cells and bone marrow mononuclear cells (BMMs) were treated with uCOCN purified from prokaryotic bacteria. Our results showed that ucOCN attenuated the proliferation of RAW264.7 cells with a concentration dependant manner by MTS assay. Scrape wounding assay revealed the decreased motility of RAW264.7 cells after ucOCN treatment. RT-qPCR results manifested the inhibitory effects of uCOCN on the expression of osteoclastic marker genes in RAW264.7 cells during inducing differentiation of RANKL. It was also observed that ucOCN inhibited the formation of multinucleated cells from RAW264.7 cells and BMMs detected by TRAP staining. The number and area of bone resorb pits were also decreased after treatment with ucOCN during their osteoclast induction by toluidine blue staining. The formation and integrity of the osteoclast actin ring were impaired by uCOCN by immunofluorescent staining. Time dependant treatment of ucOCN during osteoclastic induction demonstrated the inhibitory effects mainly occurred at the early stage of osteoclastogenesis. Signaling analysis of luciferase activity of the CRE or SRE reporter and ERK1/2 phosphorylation showed the selective inhibitor or siRNA of Gprc6a (a presumptive ucOCN receptor) could attenuate the promotion of ucOCN on CREluciferase activity. Taken together, we provided the first evidence that ucOCN had negative effects on the early differentiation and bone resorption of osteoclasts via Gprc6a. 
1 Undercarboxylated osteocalcin inhibits the early differentiation of osteoclast mediated by Gprc6a

2 Hailong Wang ${ }^{1 *}$, Jinqiao $\mathrm{Li}^{1,2^{*}}$, Zihan $\mathrm{Xu}^{1}$, Feng Wu${ }^{1}$, Hongyu Zhang ${ }^{1}$, Chao Yang ${ }^{1}$, Jian

3 Chen $^{1,3}$, Bai Ding ${ }^{1}$, Xiukun Sui ${ }^{1}$, Zhifeng Guo ${ }^{1}, Y_{\text {inghui } \text { Li }^{1 \#} \text {, Zhongquan Dai }}{ }^{1 \#}$

$4{ }^{1}$ State Key Laboratory of Space Medicine Fundamentals and Application, China Astronaut

5 Research and Training Center, Beijing, 100094, China

$6 \quad 2$ Space Engineering University, Beijing, 101416, China

$7{ }^{3}$ Department of Orthopaedics, Sir Run Run Shaw Hospital, School of Medicine, Zhejiang

8 University, Hangzhou, 310016, China

$9{ }^{*}$ These authors contributed equally to this work.

$10{ }^{*}$ Co-corresponding Author:

11 Zhonguan Dai: No.26, Beiqing Road, Haidian District, Beijing, 100094, China. E-mail address:

12 daizhq77@163.com

13 Yinghui Li: No.26, Beiqing Road, Haidian District, Beijing, 100094, China. E-mail address:

14 yinghuidd@vip.sina.com

\section{Abstract}

16 Osteocalcin $(\mathrm{OCN})$ was the most abundant noncollagen protein and considered as an endocrine

17 factor. However, the functions of Undercarboxylated osteocalcin (ucOCN) on osteoclast and

18 bone resorption are not well understood. In the present study, preosteoclast RAW264.7 cells and

19 bone marrow mononuclear cells (BMMs) were treated with ucOCN purified from prokaryotic

20 bacteria. Our results showed that ucOCN attenuated the proliferation of RAW264.7 cells with a

21 concentration dependant manner by MTS assay. Scrape wounding assay revealed the decreased

22 motility of RAW264.7 cells after ucOCN treatment. RT-qPCR results manifested the inhibitory

23 effects of ucOCN on the expression of osteoclastic marker genes in RAW264.7 cells during

24 inducing differentiation of RANKL. It was also observed that ucOCN inhibited the formation of

25 multinucleated cells from RAW264.7 cells and BMMs detected by TRAP staining. The number

26 and area of bone resorb pits were also decreased after treatment with ucOCN during their 
27 osteoclast induction by toluidine blue staining. The formation and integrity of the osteoclast actin 28 ring were impaired by ucOCN by immunofluorescent staining. Time dependant treatment of ucOCN during osteoclastic induction demonstrated the inhibitory effects mainly occurred at the early stage of osteoclastogenesis. Signaling analysis of luciferase activity of the CRE or SRE reporter and ERK1/2 phosphorylation showed the selective inhibitor or siRNA of Gprc6a (a presumptive ucOCN receptor) could attenuate the promotion of ucOCN on CRE-luciferase activity. Taken together, we provided the first evidence that ucOCN had negative effects on the early differentiation and bone resorption of osteoclasts via Gprc6a.

\section{Introduction}

Osteocalcin $(\mathrm{OCN})$ is one of the most abundant noncollagen proteins and initially considered as an osteoblast-specifically secreted biomarker of bone turnover for the clinical diagnosis (Kruse \& Kracht 1986). OCN protein consists of 46-50 amino acids according to the species, and contains three highly conserved glutamic acid residuces (Glu), which were carboxylated by a vitamin K dependent $\gamma$-glutamyl carboxylase (GGCX) (Hauschka et al. 1989). The carboxylated osteocalcin (cOCN) has a stronger affinity with hydroxyapatite (HA) in the presence of calcium ions and then deposits into the bone matrix (Hauschka \& Carr 1982; Li et al. 2016). OCN is thought to play a vital role in skeletal development. Previous studies have showed that the percentage of undercarboxylated OCN (ucOCN) was increased early and remained high during spaceflight, which means OCN maybe play an important role during bone alternation during microgravity exposure (Caillot-Augusseau et al. 2000). The exact function of OCN in bone and the whole body is still unknown (Diegel et al. 2020).

Although there were some controversial data from in vitro and in vivo studies, the dual roles of OCN in bone were presumed: 1) modulating the activity of osteoblasts and osteoclasts, 2) acting as a regulator of bone mineralization (Neve et al. 2013). The expression of OCN increased with the later stage of osteoblast differentiation and highly expressed in mature osteoblasts and preosteocytes. Contrary to expectations, OCN-deficient mice developed a phenotype characterized with higher bone mass and biomechanical properteries, but without impaired bone resorption (Ducy et al. 1996). Completed knockout of OCN resulted in significantly increased trabecular thickness, density, and volume in rat bone (Lambert et al. 2016). Overexpressing OCN in mice bone showed a relatively normal state of mineralization (Murshed et al. 2004). The exact 
57 function of $\mathrm{OCN}$ in bone homeostasis remains controversial.

58 Involvement of $\mathrm{OCN}$ in bone resorption was also investigated, especially in the regulation of 59 osteoclast recruitment, differentiation, and activity. Purified OCN from lyophilized chicken bone 60 promoted a dose dependent chemotactic response in osteoclast progenitor monocytes (Malone et

61 62 63 64 65 66 al. 1982). The bone particles (BP) from warfarin treated rat (daily subcutaneous injections of sodium warfarin 1 day after birth for 42 days) showed little OCN and less Gla content than that from normal rats. After implantation into bilateral, subcutaneous pockets of normal rat, the warfarin treated BPs were resorbed only $60 \%$ of normal BP detected by histomorphometric analysis and recruited fewer osteoclasts with decreased tartrate-resistant acid phosphatase (TRAP) activity (Glowacki \& Lian 1987; Lian et al. 1984; Liggett et al. 1994). These results suggested that OCN deficiency could inhibit the recruitment of osteoclast progenitor and its differentiation, especially carboxylated OCN, and OCN played an important role in the osteoclast development and functions. However, genetic studies demonstrated deletion of OCN resulted in unimpaired bone resorption after ovariectomy (Ducy et al. 1996). Due to the decreased GGCX activity and the low pH during bone resorption, some unincorporated cOCN and undercarboxylated $\mathrm{OCN}(\mathrm{ucOCN})$ are released into the circulation and considered as a clinical marker of bone turnover (Clemens \& Karsenty 2011; Plantalech et al. 1991) and have a concentration range of 40-228 ng/ml in mouse serum (Srivastava et al. 2000). Recently, increasing evidences indicate that $\mathrm{uCOCN}$ is an endocrine hormone which regulates energy metabolism (Lee et al. 2007), male fertility (Oury et al. 2011), muscle mass(Mera et al. 2016), brain development, and recognition (Oury et al. 2013). But the exact function and regulation model of ucOCN in bone are far away understood, especially in osteoclast and bone resorption (Manolagas 2020). Furthermore, Gprc6a, a G protein-coupled receptor class C group 6, was considered as its candidate receptor (Oury et al. 2011), but not all thought so (Rueda et al. 2016).

In the present study, we hypothesized that ucOCN could affect the maturity and function of osteoclasts. ucOCN was purified from artificially constructed prokaryotic bacteria and added to preosteoclast RAW264.7 cells and BMMs. The results showed that ucOCN decreased the proliferation, motility, differentiation, and maturation of the preosteoclast. Further reporter assay and knockdown examination demonstrated the Gprc6a mediated the negative regulation of ucOCN on osteoclast. 


\section{Materials and methods}

\section{Cells cultures}

89 All the cell lines used in the present study were obtained from Chinese National Infrastructure of

90 Cell Line Resource. RAW264.7 cells were cultured in DMEM (HyClone, USA) supplemented

91 with $10 \%$ FBS (Gibco, USA) and incubated at $37^{\circ} \mathrm{C}$ in $5 \% \mathrm{CO}_{2}$ air humidified incubator.

92 BMMs were isolated from the long bones of 6-week-old mice and maintained in $\alpha$-minimal

93 essential medium containing 10\% FBS in the presence of MCSF (10 ng/ml, Pepro Tech, USA).

94 To generate osteoclasts from BMMs, cells were plated in 24-well tissue culture plates and

95 cultured in the presence of $30 \mathrm{ng} / \mathrm{ml} \mathrm{MCSF}$ and $50 \mathrm{ng} / \mathrm{ml}$ RANKL (R\&D System, USA).

\section{The ucOCN production}

97 GST-OCN fusion protein was bacterially produced and purified by Glutathione Sepharose Fast

98 Flow column (GE Healthy Life Science, USA) according to standard procedures. OCN

99 protein/gene was then cleaved out from the GST subunit using thrombin. Its purity and

100 concentration were evaluated by ELISA (Clontech, USA).

\section{Cell migration assays}

102 Migration was measured by wound healing assay. RAW264.7 cells were plated at a density of

$1034 \times 10^{5}$ cells $/ \mathrm{ml}$ into $24-w e l l$ plates. When cells achieved $80 \%$ confluence, the plates were

104 streaked with a sterile micropipette tip. After rinsing off the released cells, the plates were

105 visualized at $10 \times$ magnification along with the streaks at time 0 and $24 \mathrm{~h}$ later. The cell migration

106 was determined by measuring wound width (in pixels) using ImageJ software (NIH, USA).

\section{Cell proliferation analysis by MTS}

108 RAW264.7 cells were seeded into a 96-well plate at a density of $1 \times 10^{5}$ cells/well, and replaced

109 medium with different concentration of $\operatorname{ucOCN}(1,10,100,1000 \mathrm{ng} / \mathrm{ml})$ after adhesion about 24

$110 \mathrm{~h}$. Then, the effect of different concentrations of ucOCN on RAW264.7 cell proliferation was

111 analyzed using the MTS assay kit according to the instructions of the manufacturer (Promega, 112 USA).

\section{RT-qPCR for gene expression analysis}


114 Total RNAs were extracted using the Trizol reagent according to the standard protocol. The 115 cDNA was synthesized from 500 ng of total RNA using PrimeScript ${ }^{\text {TM }}$ RT reagent Kit with

116 gDNA Eraser (Takara, Dalian China) according to the manufacturer's instructions, and

117 performed the analysis of gene expression using TB Green ${ }^{\mathrm{TM}} \mathrm{Ex} \mathrm{Taq}^{\mathrm{TM}}$ II (Tli RnaseH) from

118 Takara on a Roche lightCycler 96 instrument with $0.4 \mu \mathrm{M}$ gene-specific primer pairs (Table 1)

119 respectively; GAPDH amplification was used as an internal reference for each sample.

\section{TRAP Staining}

121 RAW264.7 cells were seeded in a 6-well plate with $100 \mathrm{ng} / \mathrm{ml} \mathrm{ucOCN}$ in the presence of 50

$122 \mathrm{ng} / \mathrm{ml}$ RANKL. BMMs were treated with ucOCN $(100 \mathrm{ng} / \mathrm{ml})$ in the presence of $30 \mathrm{ng} / \mathrm{ml} \mathrm{MCSF}$

123 and $50 \mathrm{ng} / \mathrm{ml}$ RANKL. The medium was replaced every alternate day. After 4 or 6 days of

124 culture, TRAP histochemical staining was performed using a leukocyte acid phosphatase kit

125 (Sigma,USA) following the instruction.

\section{Toluidine blue staining of bovine bone slices for osteoclast resorption}

127 Toluidine blue was a basic thiazine metachromatic dye with high affinity for acidic tissue

128 components and widely utilized for bone resorption pit staining. In brief, bovine slices (Beijing

129 Keruimei technology CO. LTD) were dipped in PBS and medium with 10-fold high

130 concentration of streptomycin and penicillin for $12 \mathrm{~h}$, respectively. RAW264.7 cells or BMMs

131 were seeded onto the slices at a density of $1 \times 10^{5}$ cells/well with the inducing factors or coupled

132 with ucOCN treatment. The medium was replaced every alternate day. After 10 days of culture,

133 the slices were washed with PBS followed by fixation with 4\% paraformaldehyde for 20 min.

134 After this, cells were washed twice with PBS and removed from bone slices via ultrasonication

135 in $0.25 \mathrm{M} \mathrm{NH}_{4} \mathrm{OH}$ for 3 times followed by distilled water. Resorption pit formation was stained

136 by $0.1 \%$ toluidine blue (Amersco, USA) for $10 \mathrm{~min}$ at room temperature. The slices were then

137 rinsed with distilled water more than 5 times to excluderesidues. Resorption pits are now stained

138 in dark blue and images were taken via light microscopy.

\section{Actin Ring staining}

140 Actin rings of mature osteoclasts were detected by actin filaments staining with rhodamine-

141 conjugated phalloidin (molecular probe, USA). Mature osteoclasts were formed from BMMs 
142 cultures in the presence of RANKL (50 ng/ml) and MSCF (30ng/ml). At the end of incubation

143 with ucOCN treatment for 6 days, osteoclasts were stained with rhodamine-conjugated

144 phalloidin for actin. The distribution of actin rings was visualized and the images were taken by

145 a Nikon Eclipse Ti microscope (Japan).

\section{Western Blot Analysis}

147 The cells were lysed by RIPA buffer (50 mM Tris-HCl (pH 7.4), 150 mM NaCl, 1\% NP-40, 1

$148 \mathrm{mM}$ EDTA, and $0.1 \%$ sodium dodecyl sulfate) containing protease inhibitor cocktail (Roche,

149 German). Each sample was kept on ice for $30 \mathrm{~min}$ before it was centrifuged at $13000 \mathrm{~g}$ for 30

$150 \mathrm{~min}$ at $4{ }^{\circ} \mathrm{C}$. The protein concentration of the supernatant was determined with a bicinchoninic

151 acid protein assay kit (Thermo Fisher Scientific, USA). The total proteins were subjected to

152 SDS-PAGE and then transferred to a polyvinylidene difluoride membrane (Millipore, USA). The

153 membranes were blocked with 5\% skim milk in Tris-buffered saline containing $0.1 \%$ Tween 20

154 (TBST) for $1 \mathrm{~h}$ and then incubated overnight with a primary antibody diluted in 5\% bovine

155 serum albumin at $4{ }^{\circ} \mathrm{C}$ (specific antibodies for RANK, c-Fms (Abcam, UK), P44/42

156 MAPK(REK1/2), Phospho-p44/42 MAPK(ERK1/2), GAPDH (CST, USA), Grpc6a (Santa Cruz,

157 USA)). The membranes were incubated with appropriate secondary antibodies conjugated with

158 horseradish peroxidase (CST, USA) for $2 \mathrm{~h}$ at room temperature. Signals were detected using

159 enhanced luminescence (Bio-Rad, USA) after washing three times. The intensity of the protein

160 bands was calculated using ImageJ software (NIH, USA).

\section{Luciferase reporter gene assay}

162 RAW264.7 cells were seeded into 24 -well plates at a density of with $1.5 \times 10^{5}$ cells $/ \mathrm{ml}$ and

163 cultured for $12 \mathrm{~h}$. The specific siRNA of Gprc6a (siRNA-2553

164 GCAGAAGACTAACACCAAA) was transfected into RAW264.7 cells for $24 \mathrm{~h}$ before reporter

165 transfection and ucOCN treatment. The reporter plasmid pGL4.33[luc2P/SRE/Hygro] or

166 pGL4.29 [luc2P/CRE/ Hygro] coupled with phRLTK was transfected into RAW264.7 cells

167 followed by treatment with $100 \mathrm{ng} / \mathrm{ml} \mathrm{ucOCN}, 20 \mathrm{mM}$ L-ornithine (Sigma, USA) alone or

168 combined with $30 \mu \mathrm{M}$ NPS2143 (Sigma, USA) as previous reports desrption (Rueda et al. 2016)

$16936 \mathrm{~h}$ later. The measurement of luciferase activity was performed with the Dual Luciferase

170 Reporter Assay kit (Promega, USA) following the manufactory’s instruction.

Peer] reviewing PDF | (2020:08:51673:2:0:NEW 11 Jan 2021) 


\section{Statistical Analysis}

172 Experiments were carried out independently at least three times. Results are expressed as the

173 mean \pm SEM and were compared by Student's t test (two groups) or Two-way repearted

174 measures ANOVA with Duncan's multiple comparison (multiple groups). Results were

175 considered significantly different for $\mathrm{p}<0.05$.

\section{Results}

177 Osteoclast precursors are attracted from the bone marrow to the bloodstream by chemokines and 178 recruited to bone by a variety of factors released at sites undergoing resorption and differentiated 179 into mature osteoclasts to resorb bone tissue (Boskey et al. 2003). Previous studies have 180 demonstrated that OCN could recruit and promote osteoclast differentiation, but the mechanism 181 was still not well understood, especially ucOCN. Thus, we tried to evaluate the effects of ucOCN 182 on the migration, proliferation, and differentiation of osteoclast recruitment process using 183 RAW264.7 cells. ucOCN attenuated the proliferation and migration of RAW264.7 cells

185 First of all, as the previous report (Oury et al. 2011), we purified ucOCN from an artificial E.coli 186 prokaryotic bacteria by GST-fusion method. Its purity and concentration were examined by specific ELISA kit. As shown in figure S1, no cOCN was detected by specific ELISA kit and a very low of endotoxin level was detected. The purified ucOCN was used in the following experiments.

To explore the effects of ucOCN on osteoclast proliferation, the mouse monocyte RAW264.7 cells were treated with different concentration of $\operatorname{ucOCN}(1,10,100,1000 \mathrm{ng} / \mathrm{ml})$ for $24 \mathrm{~h}, 48 \mathrm{~h}$ and $72 \mathrm{~h}$. The growth rate of the different treated cells was detected by MTS method and it was significantly inhibited by ucOCN treatment at the indicated time point as shown in Fig $1 \mathrm{~A}$. The inhibition effect of ucOCN at the lowest concentration $(1 \mathrm{ng} / \mathrm{ml})$ just lasted for $48 \mathrm{~h}$, but the inhibition effects of ucOCN at other three concentrations $(10,100,1000 \mathrm{ng} / \mathrm{ml})$ still

196 significantly occurred at $72 \mathrm{~h}$ time point. There was about $40 \%$ inhibition at $24 \mathrm{~h}$ and $48 \mathrm{~h}$, but 197 only $20-30 \%$ at $72 \mathrm{~h}$. These results indicated that ucOCN attenuated the proliferation activity of RAW264.7 cells with concentration and time-dependant pattern. 
199 The migration of RAW264.7 cells was measured in a scrape wounding assay over $24 \mathrm{~h}$ with $200 \mathrm{ucOCN}(100 \mathrm{ng} / \mathrm{ml})$ treatment or not. As shown in Fig 1B-1C, there was a higher motility in 201 untreated RAW264.7 cells than ucOCN treated cells. In the control group, the cells at the edge of 202 the steak were significantly migrated toward the middle and the width of the scribe line became 203 narrowed. But there were almost no changes in the ucOCN treated group. The above results 204 suggested that ucOCN inhibited the motility of RAW264.7 cells.

205

206

207

208

209

210

211

212

213

214

215

216

217

218

219

220

221

222

223

224

225

226

227

228

\section{ucOCN suppressed RANKL-induced osteoclastogenesis.}

ucOCN affected the osteoclastic gene expression. To clarify the effects of ucOCN on osteoclastogenesis, we examined the osteoclastic gene expression during induction in the presence of ucOCN by RT-qPCR. The preosteoclastic RAW264.7 cells were induced to osteoclastic differentiation by $50 \mathrm{ng} / \mathrm{ml}$ receptor activator of nuclear factor- $\kappa$ B ligand (RANKL), a key osteoclastogenic cytokine, without or with ucOCN $(10 \mathrm{ng} / \mathrm{ml}$ or $100 \mathrm{ng} / \mathrm{ml}$ based on the proliferation effects results) for 4 days, then lysed by Trizol for gene expression assay. As shown in Fig 2A, the marker genes (NFATc1, RANK, TRAF6, etc) of osteoclastic differentiation were significantly increased following RANKL induction, especially the upregulation of CathK and TRAP genes about several hundred folds. Compared with the ucnOCN untreated control group (CN) coupled with RANKL induction, the expression of these genes dramatically decreased about $40-60 \%$ after treatment with ucOCN accompanied with RANKL induction. The inhibition efficiency of $100 \mathrm{ng} / \mathrm{ml} \mathrm{ucOCN}$ was higher than that of $10 \mathrm{ng} / \mathrm{ml}$ (Fig. 2B) and it was reported that serum concentration of ucOCN could be $35.9 \mathrm{ng} / \mathrm{mL}$ in mice (Prats-Puig et al. 2014), So 100 $\mathrm{ng} / \mathrm{ml}$ was used in the following experiments.

Secondarily, mature TRAP positive multinucleated cells (MNCs) were assayed in RAW276.4 cells and BMMs. Cells were incubated with $100 \mathrm{ng} / \mathrm{mL}$ ucOCN in the presence of RANKL for RAW264.7 cells or in the presence of RANKL and MCSF for BMMs. RAW264.7 cells differentiated to mature TRAP-positive MNCs in the absence of ucOCN, however, fewer MNCs formation was observed in the presence of $100 \mathrm{ng} / \mathrm{ml} \mathrm{ucOCN}$ (Fig. 3A). ucOCN inhibited the formation and number of TRAP positive MNCs. In BMMs, ucOCN appeared to have similar effects. Indeed, there were no purple TRAP positive cells after treatment of MCSF or combined with ucOCN, neither mononuclear cells or multinucleated cells (Fig S2). The treatment of BMMs with RANKL and MCSF induced the formation of TRAP positive MNCs, nevertheless 
ucOCN decreased the induction effects of RANKL to osteoclast (Fig.3C).

230 We further examined the effects of ucOCN on the function of mature osteoclasts to resorb bone.

231 RAW264.7 cells were plated onto bone slices and induced with RANKL in the presence of 100

$232 \mathrm{ng} / \mathrm{mL}$ ucOCN. RANKL stimulated cells form a number of pits, which indicated successful

233 induction of RAW264.7 cells to functional bone resorption activity by RANKL. As shown in

234 Fig.3B, treatment of $100 \mathrm{ng} / \mathrm{ml} \mathrm{ucOCN}$ significantly reduced the formation of resorption pits in

235 number and areas compared with treatment with RANKL alone. Similar results were observed in

236 BMMs after induced with MCSF+RANKL combined with ucOCN (Fig.3D). During bone

237 resorption, an actin ring was formed to seal the resorption area. To investigate the effects of

$238 \mathrm{ucOCN}$ on actin ring formation, immunofluorescence staining was carried out for BMM cells

239 induced by RANKL in the presence of ucOCN. There were single cells stained with red in only

240 MCSF treatment group. Well-formed actin rings were observed in the MCSF and RANKL-

241 treated cells. After the addition of ucOCN during the progress of induction of MCSF and

242 RANKL, the number of red actin rings decreased, and the structure of formed actin rings showed

243 irregularity and shrinkage (Fig.3E). These results indicated the inhibitory effects of ucOCN on

244 the bone resorption of osteoclast and the formation and integrity of the osteoclast actin ring.

245 ucOCN affected the initial differentiation of osteoclasts.

246 The above results suggested the inhibition effects of ucOCN on osteoclast differentiation. Next,

247 we tried to explore which stage of osteoclast differentiation was affected by ucOCN. To achieve

248 this aim, ucOCN was added to the MCSF+RANKL inducing medium in the final concentration

249 of $100 \mathrm{ng} / \mathrm{mL}$ for BMM cells at different time points as indicated $\left(0,2^{\text {nd }}, 4^{\text {th }}\right.$ day) in Fig. $4 \mathrm{~A}$, then

250 some classic osteoclastic genes were detected by RT-qPCR. MNCs were assayed by TRAP

251 staining at the $6^{\text {th }}$ day. Compared with the ucOCN untreated group $(\mathrm{CN})$, the expressions of

252 RANK, TRAP, CathK, and CTR were significantly decreased about 20-50\% when ucOCN was

253 simultaneously added with inducing reagent, and only descent tendency was observed when

254 ucOCN was added at the $2^{\text {nd }}$ day or almost no effects were detected when ucOCN was added at

255 the $4^{\text {th }}$ day (Fig. 4B). The inhibition ratio of ucOCN on the expression of classic osteoclastic

256 genes at 0 day (20-50\%) was similar to that of RAW264.7 cells as shown in Fig.2B (40-60\%).

257 There was abundant of TRAP positive MNCs in the MCSF and RANKL inducing group, which

258 was markedly reduced in the ucOCN additional group at day 0 . The number of TRAP positive 
259 MNCs showed a slight decreased in the $2^{\text {nd }}$ day group and no changes occurred in the $4^{\text {th }}$ day

260 group when compared with the ucOCN untreated inducing group (Fig. 4C). It was accordant

261 with the results of gene expression. These results demonstrated that ucOCN could attenuated the

262 early differentiation of osteoclasts.

263 To further confirmation this phenomenon, RAW264.7 cells were added with $100 \mathrm{ng} / \mathrm{mL}$ ucOCN

264 for 2 days, then evaluated the expression of c-Fms (the receptor of MCSF) and RANK (the

265 receptor of RANKL) genes by RT-qPCR, western blot. As shown in Fig.4D-4F, the mRNA and

266 protein expression of c-Fms and RANK were inhibited about 30-50\% after addition of ucOCN.

267 These results suggested that ucOCN could inhibit the expression of RANK and c-Fms in

268 RAW264.7 cells.

\section{Gprc6a mediated the effects of ucOCN on osteoclasts.}

270 ucOCN promoted beta-cells proliferation and testosterone synthesis in Leydig by Gprc6a, an 271 orphan G protein-coupled receptor (Oury et al. 2011; Wei et al. 2014). Gprc6a is a cation-,

272 calcimimetic-, and OCN-sensing receptor and widely expressed in brain and peripheral tissues,

273 with the highest levels in kidney, skeletal muscle, testis and leucocyte (Wellendorph \& Brauner-

274 Osborne 2004). but it was unknown if osteoclastic lineage cells expressed Gprc6a. In this regard,

275 The expression of Gprc6a in RAW264.7 cells and BMMs were detected by RT-PCR, western

276 blot, and immunofluorescent staining method. Similar to the positive control TC-6 cells, bands of

277 the desired size were observed in the agarose gel electrophoresis of RT-PCR production and in

278 the PVDF film of protein by western blot detection from both osteoclastic lineage cells (Fig.5A,

279 5B). Immunofluorescent staining also demonstrated the expression of Gprc6a, which localized in

280 the cytoplasm membrane of RAW264.7 cells and mature osteoclast (Fig.5C).

281 Several reports have demonstrated that ucOCN could activate ERK and serum response element

282 (SRE)-luciferase reporter activity (Pi et al. 2005), a transcriptional effector CREB in Leydig

283 (Oury et al. 2011). To evaluate if Gprc6a was activated by ucOCN in osteoclastic linage cells,

284 the SRE- or CRE- (cAMP response element) luciferase reporter was transfected into RAW264.7

285 cells coupled with Renilla phRLTK vector. After transfection for $36 \mathrm{~h}$, the cells were treated with

286 ucOCN, L-ornithine (an agonist of Gprc6a) with or without $10 \mu \mathrm{M}$ NPS2143 (non-competitive

287 antagonist of Gprc6a (Faure et al. 2009), and then the transcriptional activity was measured with 
288 the Dual Luciferase Assay. To screen an optimal time point, the cells were treated for $6 \mathrm{~h}, 12 \mathrm{~h}$, 289 and $24 \mathrm{~h}$ after $36 \mathrm{~h}$ of reporter transfection, as illustrated in Fig.5D. The luciferase activity was 290 significantly increased after treatment with ucOCN at the indicated time, especially at $12 \mathrm{~h}$ 291 which was performed in the following experiment (Fig.5E). Although there was no effect of 292 NPS2143 on the luciferase activity, the promotive effects were eliminated by NPS2143 co293 treatment, which was concordance with the positive control groups of L-ornithine (Fig.5F).

294 Similar results were achieved by CRE-luciferase detection (Fig.5G). Moreover, increased 295 ERK1/2 phosphorylation was observed after $12 \mathrm{~h}$ treatment of ucOCN or L-ornithine, which was 296 also wiped out by NPS2143 (Fig. 5I). Next, a Gprc6a specific siRNA (Pi et al. 2010) was

297 transfected into RAW267.4 cells for $48 \mathrm{~h}$, followed by CRE-luciferase reporter transfection and 298 ucOCN treatment as the above description. As shown in Fig.5H, the promotion of ucOCN and L299 ornithine to CRE luciferase activity was completely ablated by the siRNA. These results implied

300 that ucOCN promoted SRE/CRE signaling and ERK1/2 phosphorylation mediated by Gprc6a.

\section{Discussion}

302 Currently, more and more evidences indicated that ucOCN is an endocrine hormone in regulating energy metabolism, brain development, muscle function, and male male fertility, but some

304 discrepancies and paradoxes emerged in the researches about the structure, physiological

305 function, receptor and clinical effects of OCN (Li et al. 2016; Moriishi et al. 2020). Early reports

306 have demonstrated that OCN promoted the recruitment and differentiation of osteoclasts, but

307 absent of OCN didn't impair the bone resorption using the ovariectomy model. Recently,

308 Moriishi et al. demonstrate that OCN is not involved in the regulation of bone quantity, glucose

309 metabolism, but required for bone quality and strength by adjusting the alignment of bone apatite

310 crystallites parallel to collagen fibrils(Moriishi et al. 2020). Till now, the exact function and

311 mechanism of OCN in bone remodeling are not well understood. In the present study, we

312 demonstrated that ucOCN inhibited the migration, proliferation, and differentiation of osteoclasts

313 in vitro.

314 To our knowledge, these results firstly showed that ucOCN had significant inhibitory effects on

315 the proliferation, migration, differentiation, and maturation of osteoclasts in both osteoclast

316 models (RAW264.7 cells and BMMs). First of all, ucOCN attenuated the proliferation and

317 motility of RAW264.7 cells with concentration and time-dependant pattern by MTS detection 
318 and scraped wounding assay (Fig.1). Secondary, osteoclast-related gene expression during

319 osteoclast induction differentiation by RANKL was inhibited by ucOCN, including the master 320 transcription factor NFATc1 (Kim \& Kim 2014) and later functional gene TRAP and CathK as

321 shown in Fig.2. Thirdly, TRAP staining and actin ring staining showed that ucOCN weakened

322 the formation of osteoclast multinuclear fusion. Meanwhile, bone resorption activity was

323 attenuated by ucOCN using toluidine blue staining of bovine bone slices (Fig.3). Interestingly,

324 we found the inhibitory effects mainly occurred in the initial stage of RANKL-inducing

325 osteoclast differentiation. As shown in Fig.4D-4F, the early regulation gene c-Fms and RANK

326 were markedly decreased after ucOCN treatment. Finally, the reporter assay system and siRNA

327 silence experiments demonstrated Gprc6a mediated effects of ucOCN on osteoclasts (Fig.5). All

328 these data demonstrated that ucOCN had a significant inhibitory effect on the differentiation and

329 maturation of osteoclasts and bone resorption.

330 Bone reconstruction with bone formation by osteoblast and bone resorption by osteoclast ensured

331 the continuous renewal of the body skeletal and maintained its homeostasis. The

332 chemoattractants responsible for the mobilization of osteoclast precursors were derived from the

333 bone matrix (Malone et al. 1982). OCN was mainly produced by osteoblasts with its

334 carboxylated form and stored in the bone matrix (Al Rifai et al. 2017; Hauschka et al. 1989).

335 Osteoblast also released some ucOCN because of the decrease of GGCX activity or insufficiency

336 of vitamin K supplement (Shiba et al. 2014). OCN-deficiency BP recruited fewer osteoclast

337 progenitors and formed osteoclasts with decreased TRAP activity (Glowacki et al. 1991). OCN

338 fragments, purified from bovine bone, influenced osteoclast maturation, especially in the late

339 stage of osteoclast differentiation (Ishida \& Amano 2004). No abnormalities in bone size,

340 morphology, and mineralization were observed in the warfarin-maintained rat bones with only

$3412 \%$ of control OCN levels (Price \& Williamson 1981). However, another study showed that rats

342 maintained for 8 months with warfarin were characterized by complete fusion of the proximal

343 tibial growth and cessation of longitudinal growth, which might be caused by the decrease of

344 bone OCN (Price et al. 1982). The OCN used in the early studies in the 1980s and 1990s was

345 extracted from animal bones by EDTA method, which included the main component of cOCN

346 and contained some undercarboxylated and fragment OCN. Our results suggested that ucOCN

347 had some negative effects on osteoclasts, which implied the promotion effects of OCN might

348 mainly come from cOCN. Recently, Ferron reported that the osteoblast specific knockout of 
349 GGCX had no effect on bone mass and bone turnover parameters (Ferron et al. 2015). The

350 GGCX specific knockout or warfarin treatment also decreased the OCN content in bone tissue,

351 which might attenuate the promotion of $\mathrm{cOCN}$ on osteoclasts. The low $\mathrm{pH}$ in the resorption

352 lacuna could decarboxylate the deposited $\mathrm{cOCN}$ and ucOCN was released into the bloodstream

353 (Lacombe et al. 2013). Combined with previous reports about the positive effects of cOCN on

354 the chemotaxis, adhesion, and differentiation of osteoclasts, it was presumed that the balance

355 between carboxylated and undercarboxylated OCN is important for bone metabolism

356 homeostasis. This inhibition effect may also act as a brake for the energy regulation endocrine

357 function of bone. As previous reports, ucOCN increased beta-cell proliferation and insulin

358 secretion (Lee et al. 2007). In turn, insulin signaling in osteoblast promoted the osteoclast ability

359 to acidify the bone extracellular matrix, which decarboxylated cOCN into ucOCN and favored

360 whole body glucose homeostasis (Ferron et al. 2010). Bone-derived ucOCN and pancreatic $\beta$ -

361 cells insulin formed a positive feed-forward regulation loop in glucose regulation. Our results

362 may provide a negative regulation of this cross talk between OCN and insulin, like its negative

363 regulator DLK1 in pancreatic $\beta$-cells (Abdallah et al. 2015).

364 Several studies had shown that the beneficial effects of ucOCN were ameliorated or absent in

365 Gprc6a KO mice, which implicated the class C GPCR (Gprc6a) as a target receptor for ucOCN

366 (Pi et al. 2011), but other KO mice displayed a subtler phenotype with no evidence of impaired

367 glucose handling and insulin sensitivity (Jorgensen et al. 2019; Smajilovic et al. 2013).

368 Moreover, Rueda et al. showed evidence that Gprc6a was not a direct ligand for ucOCN, rather

369 than an indirect requirement for the effects of OCN (Rueda et al. 2016). L-amino-sensing,

370 activation of cAMP signaling and ERK1/2 phosphorylation properties of Gprc6a were well

371 characterized, so does the SRE activity. Small molecules, such as NPS-2143, which could bind

372 to the 7TM domain of the receptor, acted as negative allosteric modulators of Gprc6a (Faure et

373 al. 2009). Similar to L-ornithine, ucOCN treatment significantly increased the SRE- or CRE-

374 luciferase activity, and ERK1/2 phosphorylation in a NPS2143-sensitive manner.

375 Downregulation of Gprc6a by specific siRNA also attenuated the promotion of ucOCN on CRE-

376 luciferase. These results indicated a Gprc6a-mediated mechanism of ucOCN effects on

377 osteoclasts (Fig5).

378 The downstream signaling of Gprc6a on the inhibition of osteoclasts by ucOCN should be 
379 further explored. Our results showed the increased ERK1/2 phosphorylation, which was reported

380 to be a positive regulation on osteoclast mature (Soysa et al. 2012), and early reports supported

381 that the MEK/ERK pathway negatively regulates osteoclastogenesis, while the p38 pathway does

382 so positively (Hotokezaka et al. 2002). By the way, the role of ucOCN on osteoclasts was similar

383 to the hormone calcitonin, which stimulated cAMP production and inhibited osteoclastic bone

384 resorption (Yang \& Kream 2008).

\section{Conclusions}

386

387

388

389

390

391

392

393

394

395

396

397

398

399

400

401

402

403

404

405

406

407

408

409

Taken together, we firstly provided evidences that ucOCN had negative effects on the proliferation, migration, early differentiation, and bone resorption of osteoclast, which was mediated by Gprc6a. This functional pattern could sever as a negative regulation mechanism for serious bone resorption during bone remodeling and for the feed-forward loop of OCN-insulin on whole body glucose homeostasis and presumed that the balance between carboxylated and undercarboxylated OCN might play an important role in bone remodeling, but the detail mechanism should be further explored. The increase of ucOCN percentage during spaceflight maybe a negative feedback to prevent the further bone resorption. For the difficult realization by current technology, our notion was a deficiency of evidence from in vivo experiments.

\section{References:}

Abdallah BM, Ditzel N, Laborda J, Karsenty G, and Kassem M. 2015. DLK1 Regulates WholeBody Glucose Metabolism: A Negative Feedback Regulation of the Osteocalcin-Insulin Loop. Diabetes 64:3069-3080. 10.2337/db14-1642

Al Rifai O, Chow J, Lacombe J, Julien C, Faubert D, Susan-Resiga D, Essalmani R, Creemers JW, Seidah NG, and Ferron M. 2017. Proprotein convertase furin regulates osteocalcin and bone endocrine function. J Clin Invest 127:4104-4117. 10.1172/JCI93437

Boskey AL, Moore DJ, Amling M, Canalis E, and Delany AM. 2003. Infrared analysis of the mineral and matrix in bones of osteonectin-null mice and their wildtype controls. $J$ Bone Miner Res 18:1005-1011. 10.1359/jbmr.2003.18.6.1005

Caillot-Augusseau A, Vico L, Heer M, Voroviev D, Souberbielle JC, Zitterman A, Alexandre C, and Lafage-Proust MH. 2000. Space flight is associated with rapid decreases of undercarboxylated osteocalcin and increases of markers of bone resorption without changes in their circadian variation: observations in two cosmonauts. Clin Chem 46:1136-1143. 
410 Clemens TL, and Karsenty G. 2011. The osteoblast: an insulin target cell controlling glucose homeostasis. J Bone Miner Res 26:677-680. 10.1002/jbmr.321

412 Diegel CR, Hann S, Ayturk UM, Hu JCW, Lim K-e, Droscha CJ, Madaj ZB, Foxa GE, Izaguirre

413

414

415

416

417

418

419

420

421

422

423

424

425

426

427

428

429

430

431

432

433

434

435

436

437

438

439

440

441

442 I, Transgenics Core VAIVa, Paracha N, Pidhaynyy B, Dowd TL, Robling AG, Warman ML, and Williams BO. 2020. An osteocalcin-deficient mouse strain without endocrine abnormalities. PLOS Genetics 16:e1008361. 10.1371/journal.pgen.1008361

Ducy P, Desbois C, Boyce B, Pinero G, Story B, Dunstan C, Smith E, Bonadio J, Goldstein S, Gundberg C, Bradley A, and Karsenty G. 1996. Increased bone formation in osteocalcindeficient mice. Nature 382:448-452. 10.1038/382448a0

Faure H, Gorojankina T, Rice N, Dauban P, Dodd RH, Bräuner-Osborne H, Rognan D, and Ruat M. 2009. Molecular determinants of non-competitive antagonist binding to the mouse GPRC6A receptor. Cell Calcium 46:323-332. https://doi.org/10.1016/j.ceca.2009.09.004

Ferron M, Lacombe J, Germain A, Oury F, and Karsenty G. 2015. GGCX and VKORC1 inhibit osteocalcin endocrine functions. J Cell Biol 208:761-776. 10.1083/jcb.201409111

Ferron M, Wei J, Yoshizawa T, Ducy P, and Karsenty G. 2010. An ELISA-based method to quantify osteocalcin carboxylation in mice. Biochem Biophys Res Commun 397:691-696. 10.1016/j.bbrc.2010.06.008

Glowacki J, and Lian JB. 1987. Impaired recruitment and differentiation of osteoclast progenitors by osteocalcin-deplete bone implants. Cell Differ 21:247-254.

Glowacki J, Rey C, Glimcher MJ, Cox KA, and Lian J. 1991. A role for osteocalcin in osteoclast differentiation. J Cell Biochem 45:292-302. 10.1002/jcb.240450312

Hauschka PV, and Carr SA. 1982. Calcium-dependent alpha-helical structure in osteocalcin. Biochemistry 21:2538-2547.

Hauschka PV, Lian JB, Cole DE, and Gundberg CM. 1989. Osteocalcin and matrix Gla protein: vitamin K-dependent proteins in bone. Physiol Rev 69:990-1047.

Hotokezaka H, Sakai E, Kanaoka K, Saito K, Matsuo K, Kitaura H, Yoshida N, and Nakayama K. 2002. U0126 and PD98059, specific inhibitors of MEK, accelerate differentiation of RAW264.7 cells into osteoclast-like cells. J Biol Chem 277:47366-47372. 10.1074/jbc.M208284200

Ishida M, and Amano S. 2004. Osteocalcin fragment in bone matrix enhances osteoclast maturation at a late stage of osteoclast differentiation. J Bone Miner Metab 22:415-429. $10.1007 / \mathrm{s} 00774-004-0503-5$

Jorgensen CV, Gasparini SJ, Tu J, Zhou H, Seibel MJ, and Brauner-Osborne H. 2019. Metabolic 
443

444

445

446

447

448

449

450

451

452

453

454

455

456

457

458

459

460

461

462

463

464

465

466

467

468

469

470

471

472

473

474

475

and skeletal homeostasis are maintained in full locus GPRC6A knockout mice. Sci Rep 9:5995. 10.1038/s41598-019-41921-8

Kim JH, and Kim N. 2014. Regulation of NFATc1 in Osteoclast Differentiation. J Bone Metab 21:233-241. 10.11005/jbm.2014.21.4.233

Kruse K, and Kracht U. 1986. Evaluation of serum osteocalcin as an index of altered bone metabolism. Eur J Pediatr 145:27-33.

Lacombe J, Karsenty G, and Ferron M. 2013. In vivo analysis of the contribution of bone resorption to the control of glucose metabolism in mice. Mol Metab 2:498-504. 10.1016/j.molmet.2013.08.004

Lambert LJ, Challa AK, Niu A, Zhou L, Tucholski J, Johnson MS, Nagy TR, Eberhardt AW, Estep PN, Kesterson RA, and Grams JM. 2016. Increased trabecular bone and improved biomechanics in an osteocalcin-null rat model created by CRISPR/Cas9 technology. Dis Model Mech 9:1169-1179. 10.1242/dmm.025247

Lee NK, Sowa H, Hinoi E, Ferron M, Ahn JD, Confavreux C, Dacquin R, Mee PJ, McKee MD, Jung DY, Zhang Z, Kim JK, Mauvais-Jarvis F, Ducy P, and Karsenty G. 2007. Endocrine regulation of energy metabolism by the skeleton. Cell 130:456-469. 10.1016/j.cell.2007.05.047

Li J, Zhang H, Yang C, Li Y, and Dai Z. 2016. An overview of osteocalcin progress. J Bone Miner Metab 34:367-379. 10.1007/s00774-015-0734-7

Lian JB, Tassinari M, and Glowacki J. 1984. Resorption of implanted bone prepared from normal and warfarin-treated rats. $J$ Clin Invest 73:1223-1226. 10.1172/JCI111308

Liggett WHJ, Lian JB, Greenberger JS, and Glowacki J. 1994. Osteocalcin promotes differentiation of osteoclast progenitors from murine long-term bone marrow cultures. $J$ Cell Biochem 55:190-199. 10.1002/jcb.240550206

Malone JD, Teitelbaum SL, Griffin GL, Senior RM, and Kahn AJ. 1982. Recruitment of osteoclast precursors by purified bone matrix constituents. J Cell Biol 92:227-230.

Manolagas SC. 2020. Osteocalcin promotes bone mineralization but is not a hormone. PLOS Genetics 16:e1008714. 10.1371/journal.pgen.1008714

Mera P, Laue K, Wei J, Berger JM, and Karsenty G. 2016. Osteocalcin is necessary and sufficient to maintain muscle mass in older mice. Mol Metab 5:1042-1047. 10.1016/j.molmet.2016.07.002

Moriishi T, Ozasa R, Ishimoto T, Nakano T, Hasegawa T, Miyazaki T, Liu W, Fukuyama R, Wang Y, Komori H, Qin X, Amizuka N, and Komori T. 2020. Osteocalcin is necessary 
476

477

478

479

480

481

482

483

484

485

486

487

488

489

490

491

492

493

494

495

496

497

498

499

500

501

502

503

504

505

506

507

508

509

for the alignment of apatite crystallites, but not glucose metabolism, testosterone synthesis, or muscle mass. PLOS Genetics 16:e1008586. 10.1371/journal.pgen.1008586

Murshed M, Schinke T, McKee MD, and Karsenty G. 2004. Extracellular matrix mineralization is regulated locally; different roles of two gla-containing proteins. J Cell Biol 165:625630. $10.1083 /$ jcb.200402046

Neve A, Corrado A, and Cantatore FP. 2013. Osteocalcin: skeletal and extra-skeletal effects. $J$ Cell Physiol 228:1149-1153. 10.1002/jcp.24278

Oury F, Khrimian L, Denny CA, Gardin A, Chamouni A, Goeden N, Huang YY, Lee H, Srinivas P, Gao XB, Suyama S, Langer T, Mann JJ, Horvath TL, Bonnin A, and Karsenty G. 2013. Maternal and offspring pools of osteocalcin influence brain development and functions. Cell 155:228-241. 10.1016/j.cell.2013.08.042

Oury F, Sumara G, Sumara O, Ferron M, Chang H, Smith CE, Hermo L, Suarez S, Roth BL, Ducy P, and Karsenty G. 2011. Endocrine regulation of male fertility by the skeleton. Cell 144:796-809. 10.1016/j.cell.2011.02.004

Pi M, Faber P, Ekema G, Jackson PD, Ting A, Wang N, Fontilla-Poole M, Mays RW, Brunden KR, Harrington JJ, and Quarles LD. 2005. Identification of a novel extracellular cationsensing G-protein-coupled receptor. J Biol Chem 280:40201-40209. 10.1074/jbc.M505186200

Pi M, Wu Y, and Quarles LD. 2011. GPRC6A mediates responses to osteocalcin in beta-cells in vitro and pancreas in vivo. J Bone Miner Res 26:1680-1683. 10.1002/jbmr.390

Pi M, Zhang L, Lei SF, Huang MZ, Zhu W, Zhang J, Shen H, Deng HW, and Quarles LD. 2010. Impaired osteoblast function in GPRC6A null mice. J Bone Miner Res 25:1092-1102. 10.1359/jbmr.091037

Plantalech L, Guillaumont M, Vergnaud P, Leclercq M, and Delmas PD. 1991. Impairment of gamma carboxylation of circulating osteocalcin (bone gla protein) in elderly women. $J$ Bone Miner Res 6:1211-1216. 10.1002/jbmr.5650061111

Prats-Puig A, Osiniri I, Soriano-Rodriguez P, Carreras-Badosa G, Bunuel-Alvarez JC, VilaPablos C, de Zegher F, Ibanez L, Bassols J, and Lopez-Bermejo A. 2014. Undercarboxylated osteocalcin relates to cardiovascular risk markers in offspring of families with metabolic syndrome. Atherosclerosis 233:272-277. 10.1016/j.atherosclerosis.2014.01.002

Price PA, and Williamson MK. 1981. Effects of warfarin on bone. Studies on the vitamin Kdependent protein of rat bone. J Biol Chem 256:12754-12759.

Price PA, Williamson MK, Haba T, Dell RB, and Jee WS. 1982. Excessive mineralization with 
510

511

512

513

514

515

516

517

518

519

520

521

522

523

524

525

526

527

528

529

530

531

532

533

534

535

536

537 growth plate closure in rats on chronic warfarin treatment. Proc Natl Acad Sci US A 79:7734-7738.

Rueda P, Harley E, Lu Y, Stewart GD, Fabb S, Diepenhorst N, Cremers B, Rouillon M-H, Wehrle I, Geant A, Lamarche G, Leach K, Charman WN, Christopoulos A, Summers RJ, Sexton PM, and Langmead CJ. 2016. Murine GPRC6A Mediates Cellular Responses to L-Amino Acids, but Not Osteocalcin Variants. PLoS One 11:e146846. 10.1371/journal.pone.0146846

Shiba S, Ikeda K, Azuma K, Hasegawa T, Amizuka N, Horie-Inoue K, and Inoue S. 2014. gamma-Glutamyl carboxylase in osteoblasts regulates glucose metabolism in mice. Biochem Biophys Res Commun 453:350-355. 10.1016/j.bbrc.2014.09.091

Smajilovic S, Clemmensen C, Johansen LD, Wellendorph P, Holst JJ, Thams PG, Ogo E, and Brauner-Osborne H. 2013. The L-alpha-amino acid receptor GPRC6A is expressed in the islets of Langerhans but is not involved in L-arginine-induced insulin release. Amino Acids 44:383-390. 10.1007/s00726-012-1341-8

Soysa NS, Alles N, Aoki K, and Ohya K. 2012. Osteoclast formation and differentiation: an overview. J Med Dent Sci 59:65-74.

Srivastava AK, Castillo G, Wergedal JE, Mohan S, and Baylink DJ. 2000. Development and application of a synthetic peptide-based osteocalcin assay for the measurement of bone formation in mouse serum. Calcif Tissue Int 67:255-259. 10.1007/s002230001109

Wei J, Hanna T, Suda N, Karsenty G, and Ducy P. 2014. Osteocalcin promotes beta-cell proliferation during development and adulthood through Gprc6a. Diabetes 63:1021-1031. $10.2337 / \mathrm{db} 13-0887$

Wellendorph P, and Brauner-Osborne H. 2004. Molecular cloning, expression, and sequence analysis of GPRC6A, a novel family C G-protein-coupled receptor. Gene 335:37-46. 10.1016/j.gene.2004.03.003

Yang M, and Kream BE. 2008. Calcitonin induces expression of the inducible cAMP early repressor in osteoclasts. Endocrine 33:245-253. 10.1007/s12020-008-9092-8 
Table $\mathbf{1}$ (on next page)

the primers list 
Table 1 the primers list

\begin{tabular}{|l|l|l|}
\hline Gene name & \multicolumn{1}{|c|}{ Forward } & \multicolumn{1}{c|}{ reverse } \\
\hline c-Fms & TCTTACGCAAAACGGTCTACTTC & CCAATTTTATCTGTGGGGGC \\
\hline RANK & TTGCTTCCCTGCTGGATTAG & AAGACGGTGCTGGAGTCTGT \\
\hline TRAF6 & TTGCACATTCAGTGTTTTTGG & TGCAAGTGTCGTGCCAAG \\
\hline NFATc1 & AGATACCACCTTTCCGCAAC & TAATTGGAACATTGGCAGGA \\
\hline TRAP & GCGACCATTGTTAGCCACATACG & CGTTGATGTCGCACAGAGGGAT \\
\hline CathK & GCGTTGTTCTTATTCCGAGC & CAGCAGAGGTGTGTACTATG \\
\hline Tcirg1 & CCATATCCCTTTGGCATTGA & GAGAAAGCTCAGGTGGTTCG \\
\hline CLC7 & GTCCTTCAGCCTCAGTCG & ACACAGCGTCTAATCACAAC \\
\hline MMP9 & GCTGACTACGATAAGGACGGCA & GCGGCCCTCAAAGATGAACGG \\
\hline CTR & TGGTGCGGCGGGATCCTATAAGT & AGCGTAGGCGTTGCTCGTCG \\
\hline GPRC6A & GCTCGAGACTGCAAGAAACC & TGAAGGCCAGAACTGTGATG \\
\hline GAPDH & ACTCCACTCACGGCAAATTCA & GGCCTCACCCCATTTGATG \\
\hline
\end{tabular}


Figure 1

The effects of ucOCN on the proliferative activity and migration of RAW264.7.

Cells were treated with ucOCN for different times and measured by MTS for its proliferation (A). The migration of RAW264.7 cells were measured by a scrape wounding assay over $24 \mathrm{~h}$ (B) and its corresponding distance statistical results (C). $* P<0.05, * * P<0.01, n \geq 3$, VS. 0 $\mathrm{ng} / \mathrm{ml}$ group or $\mathrm{CN}$.
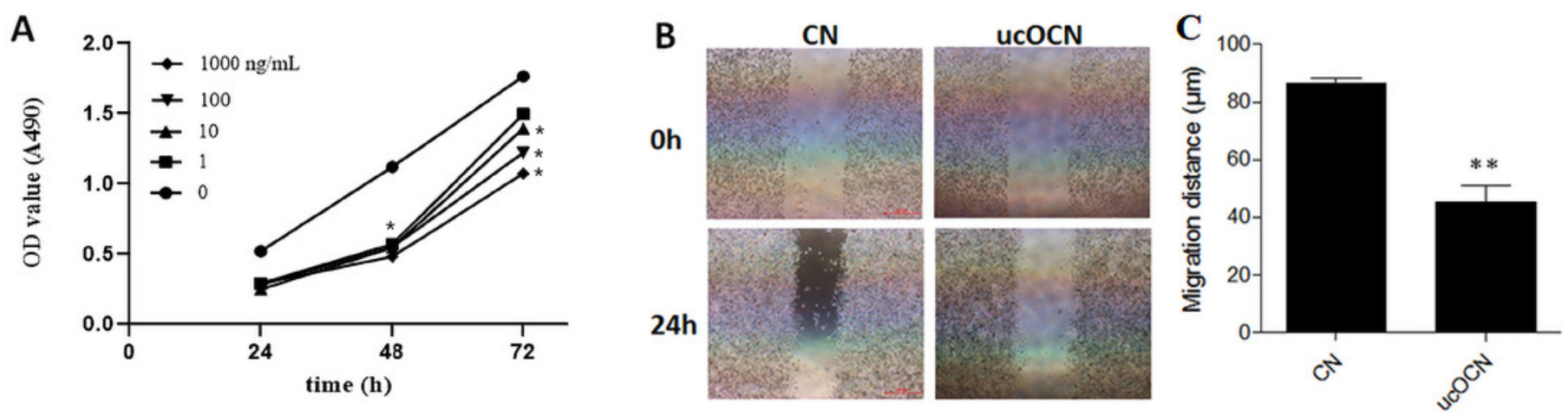
Figure 2

ucOCN inhibited osteoclast-related gene expression of RAW264.7 cells induced by RANKL.

RANKL successfully induced the increased expression of osteoclast genes (A), which was significantly attenuated by ucOCN treatment (B). $* \mathrm{P}<0.05, * * \mathrm{P}<0.01, \mathrm{n} \geq 3$ VS. CN (the uCOCN untreated control group).
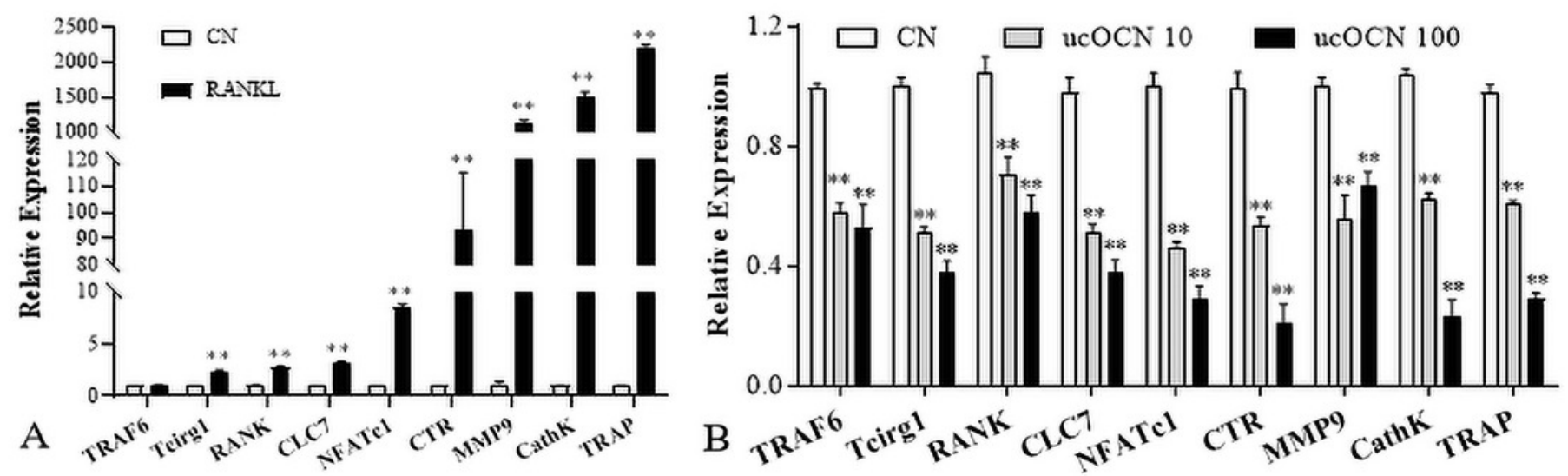

A

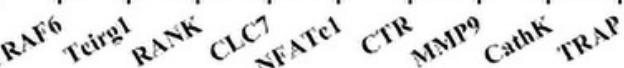

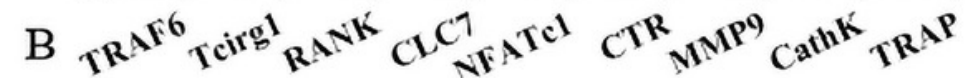




\section{Figure 3}

UCOCN decreased the TRAP positive MNCs, bone resorption activity and actin ring formation.

The first column was the negative control. Cells were incubated with ucOCN $(100 \mathrm{ng} / \mathrm{ml})$ in the presence of RANKL (50 ng/ml) for RAW264.7 cells or in the presence of RANKL $(50 \mathrm{ng} / \mathrm{ml}$ ) and MCSF (30 ng/ml) for BMMs for $4-6$ days, then performed for TRAP staining (4 days) of RAW264.7 cells (A) and BMMs (C). Toluidine blue staining (6 days) of RAW264.7 cells (B) and BMMs (D). Actin ring staining of differentiated BMMs (E) Image was taken by fluorescence microscope with a $4 \times$ object lens. 


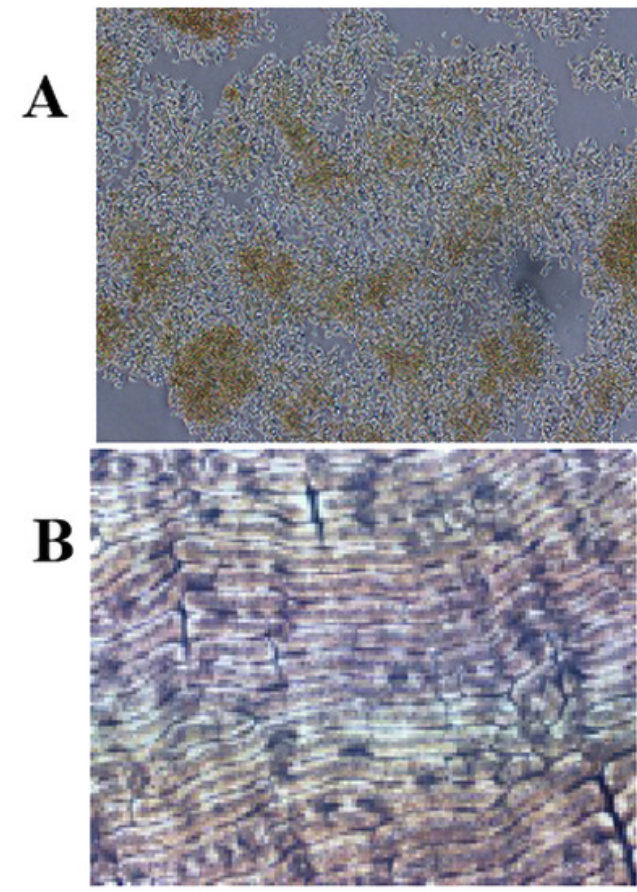

Medium

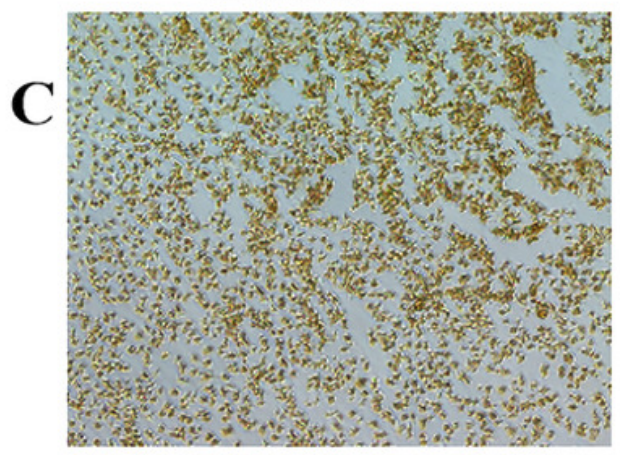

D
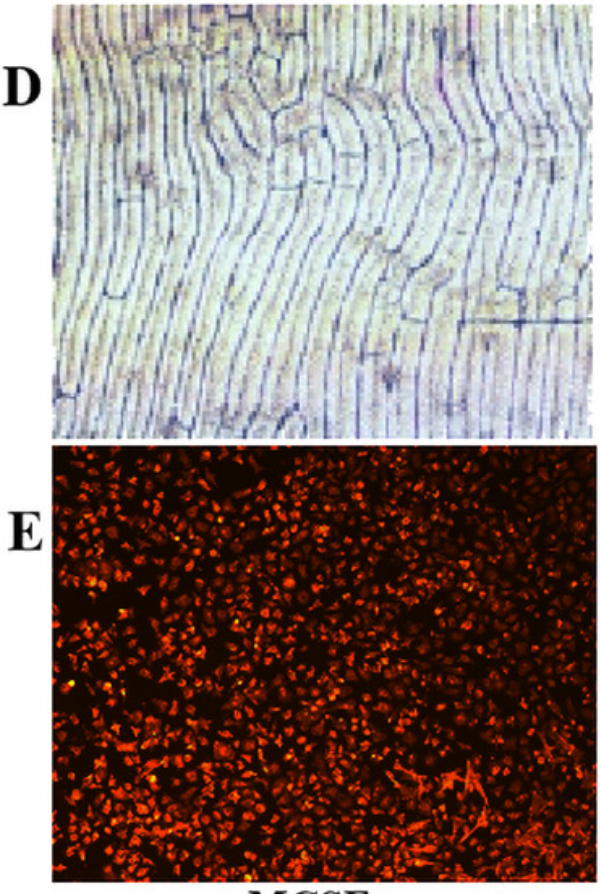

MCSF

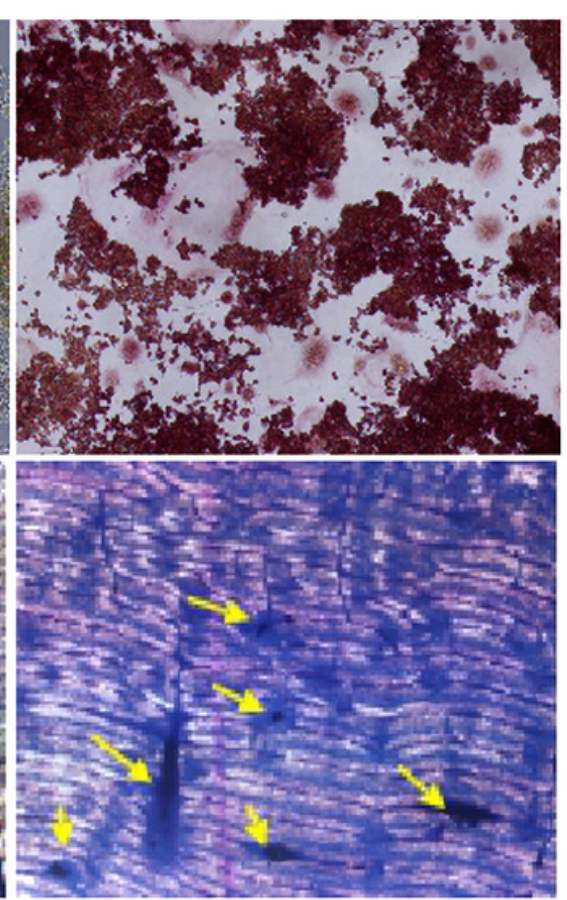

RANKL
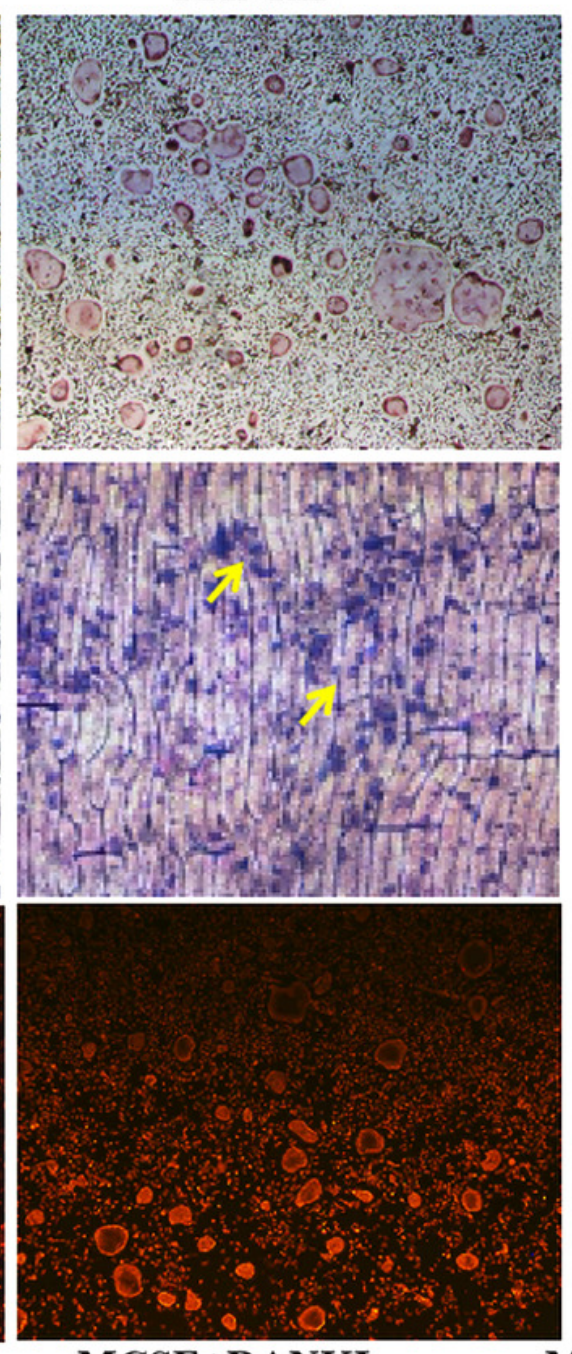

MCSF +RANKL

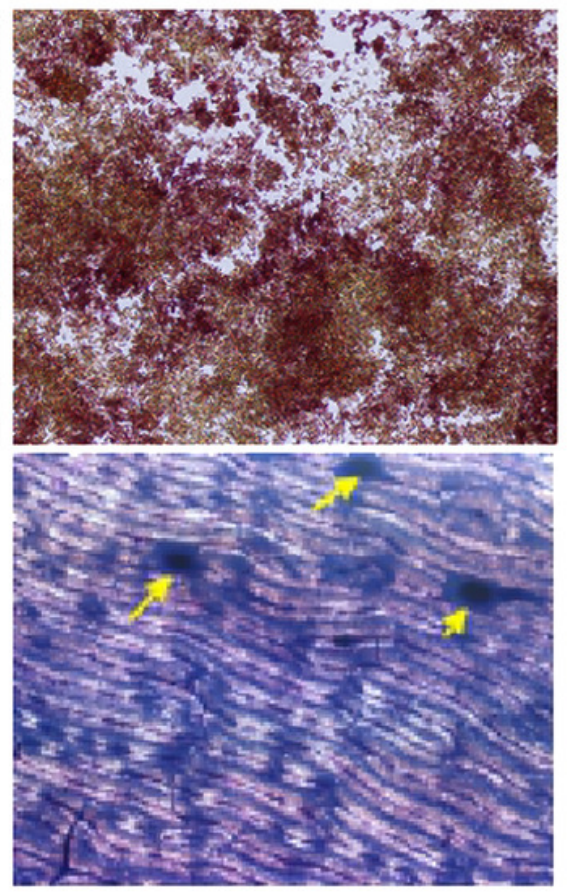

RANKL+ucOCN 100
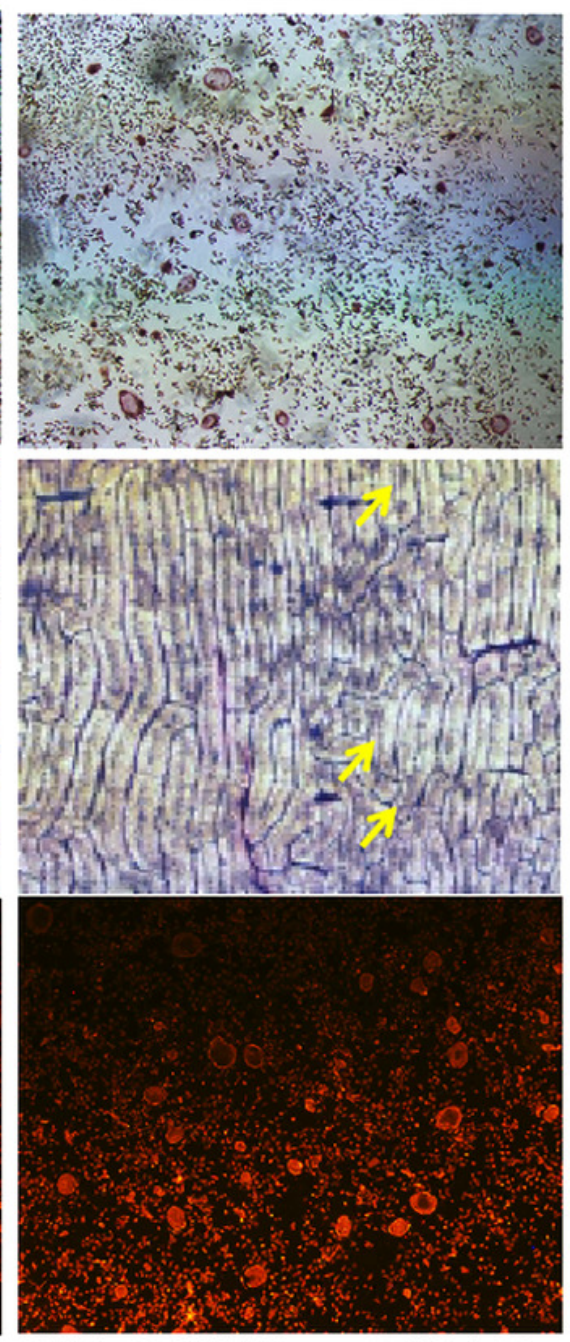

MCSF + RANKL+ uCOCN 100 


\section{Figure 4}

ucOCN inhibited the early differentiation of BMMs.

Experimental design and operation chart (A). RT-qPCR detected osteoclast-relate gene expression (B). TRAP staining (C). RAW264.7 cells were treated with ucOCN for 2 day without inducing factor RANKL, then performed RT-qPCR detection for the mRNA expression (D), Western blot detection for the protein expression (E) of c-Fms and RANK and its gray analysis of protein bands $(\mathrm{F}) . * \mathrm{P}<0.05, * * \mathrm{P}<0.01, \mathrm{n} \geq 3, \mathrm{VS}$. CN (the ucOCN untreated control group).

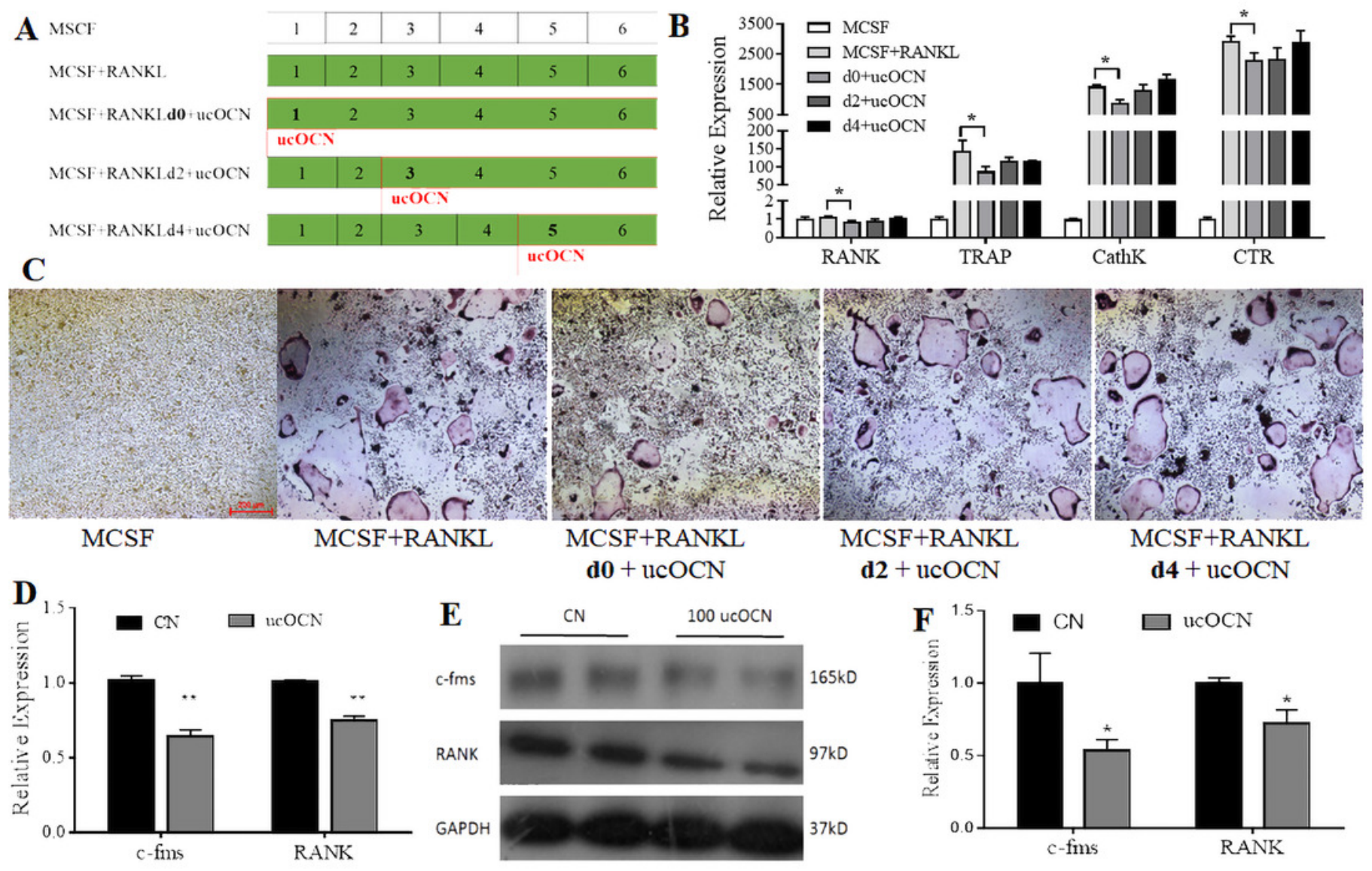




\section{Figure 5}

Gprc6a was expressed in osteoclast and mediated the effects of ucOCN on osteoclast.

mRNA (A) and protein (B) expression detection in TC-6(beta cell as positive control),

RAW264.7 cells and BMMs. immunofluorescent staining results of Gprc6a in RAW264.7 cells and mature osteoclast from BMMs (C). SRE- and CRE-miniP (minimal promoter)-luciferase reporter assay system (D) detected the SRE activity in different time with uCOCN addition (E). SRE (F) and CRE (G) activity after treatment uCOCN for 12 after $36 \mathrm{~h}$ reporter transfection in RAW264.7 cells. Gprc6a siRNA was transfected into RAW264.7 cells for $48 \mathrm{~h}$ before CRE luciferase transfection, uCOCN treatment, and reporter detection as above description $(\mathrm{H})$. Western blot detection of the phosphorylated ERK $12 \mathrm{~h}$ after uCOCN treatment and its band gray analysis (I). $* \mathrm{P}<0.05, * * \mathrm{P}<0.01, \mathrm{n} \geq 3, \mathrm{VS}$. CN (the control group without treatments). 

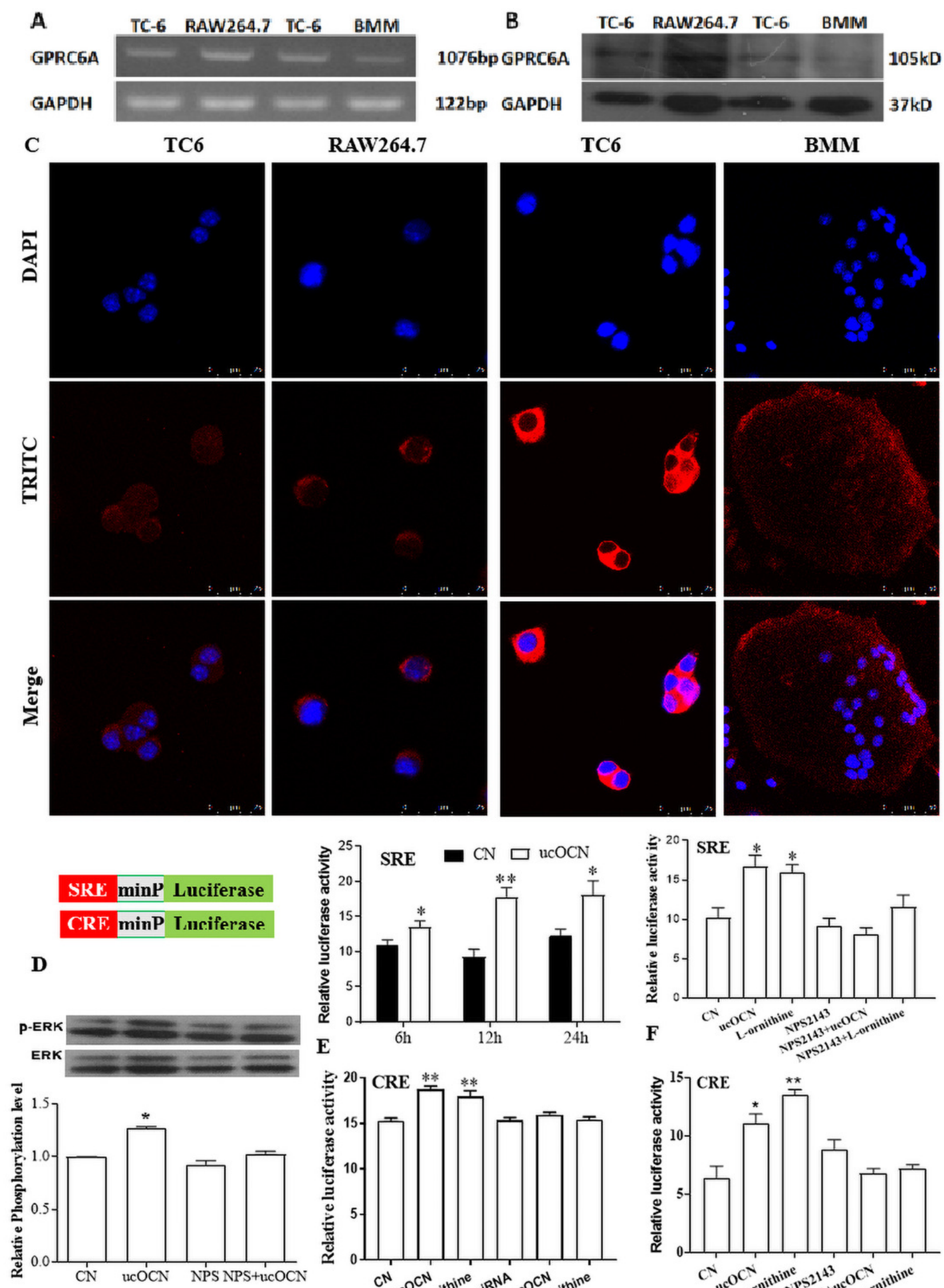

I
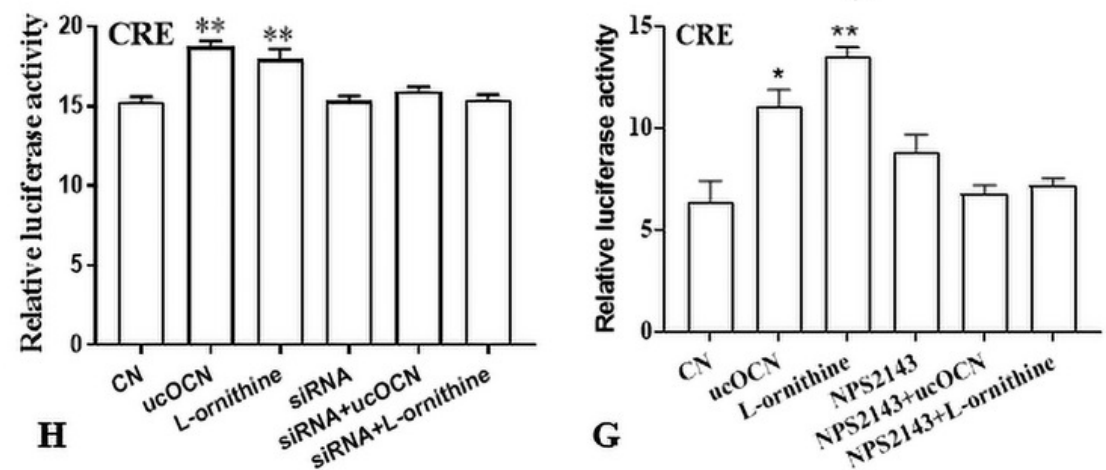